\title{
On $k$-coverage in a mostly sleeping sensor network
}

\author{
Santosh Kumar · Ten H. Lai · József Balogh
}

Published online: 23 October 2006

(C) Springer Science + Business Media, LLC 2006

\begin{abstract}
Sensor networks are often desired to last many times longer than the active lifetime of individual sensors. This is usually achieved by putting sensors to sleep for most of their lifetime. On the other hand, event monitoring applications require guaranteed $k$-coverage of the protected region at all times. As a result, determining the appropriate number of sensors to deploy that achieves both goals simultaneously becomes a challenging problem. In this paper, we consider three kinds of deployments for a sensor network on a unit square- $\mathrm{a} \sqrt{n} \times \sqrt{n}$ grid, random uniform (for all $n$ points), and Poisson (with density $n$ ). In all three deployments, each sensor is active with probability $p$, independently from the others. Then, we claim that the critical value of the function $n p \pi r^{2} / \log (n p)$ is 1 for the event of $k$-coverage of every point. We also provide an upper bound on the window of this phase transition. Although the conditions for the three deployments are similar, we obtain sharper bounds for the
\end{abstract}

An earlier version of this paper appeared in the Tenth Annual International Conference on Mobile Computing and Networking (ACM MobiCom), September 25-October 1, 2004, Philadelphia, PA. Major differences from the conference version are in Sections 5, 6, and 7.

\footnotetext{
S. Kumar $(\bowtie)$

Department of Computer Science, University of Memphis, Memphis, TN, USA 38152-3240

e-mail: santosh.kumar@memphis.edu

T. H. Lai

Department of Computer Science and Engineering, Ohio State University, Columbus, OH, USA 43210

e-mail: lai.1@osu.edu

J. Balogh

Department of Mathematical Sciences, University of Illinois,

Urbana, IL, USA 61801

e-mail: jobal@math.uiuc.edu
}

random deployments than the grid deployment, which occurs due to the boundary condition. In this paper, we also provide corrections to previously published results. Finally, we use simulation to show the usefulness of our analysis in real deployment scenarios.

Keywords Sensor network - Deterministic deployment . Random deployment - Coverage · Connectivity · Power management

\section{Introduction}

This work was motivated by a fundamental problem currently facing the deployment of sensor networks for event monitoring applications such as fire detection, radiation detection, and intrusion detection. Given an area to be protected, how many sensors should be deployed so that every point in the region is continuously covered by at least $k$ active sensors, and given that the network must last for a specified length of time? This problem is challenging because individual motes (a popular device that hosts sensors [10]) can last only 100120 hours (4-5 days) on a pair of AA batteries in the fully active mode [5], while the network is desired to last for several months. It is well known that when the motes are in the sleeping mode, they consume only $0.1 \%$ of the energy consumed in the active mode. ${ }^{1}$ A natural approach to extend the network's lifetime is, therefore, to let sensor nodes sleep in turns. The fraction of time that a node remains active is called its duty cycle.

Three issues need to be resolved before the above approach becomes feasible. First, a method is needed to compute the

\footnotetext{
${ }^{1}$ Experimental data shows that motes can last for more than a year on a $1 \%$ duty cycle [5].
} 
required duty cycle for individual nodes, given the longevity requirement of the network. Second, given a duty cycle, each node needs to determine when to be active and when to enter the sleeping mode. Third, and the most difficult, we need a method to determine the appropriate number of sensor nodes to be deployed that simultaneously achieves the desired goals of longevity and continuous $k$-coverage. This is a nontrivial problem because on the one hand, the economics dictates minimizing the number of sensors needed in the deployment, and on the other hand, it is essential to have enough of them deployed that will guarantee a desired quality of monitoring for a long enough period of time. The lack of a sound method for computing the appropriate number of sensors needed often becomes a cause for concern in actual deployments. Indeed, it is such a concern that has motivated our work.

Let us address the easier problems of determining an appropriate duty cycle and the sleeping schedule. Consider the following sleeping scheme, which we call Randomized Independent Sleeping (RIS): Time is divided into periods. Although the length of period is same for all nodes, the start time of a period need not be synchronized. At the beginning of a period, each sensor node independently decides whether to remain awake for this period (with probability $p$ ) or go to sleep (with probability $(1-p)$ ). With this approach, the expected lifetime of the network will increase by a factor close to $1 / p$. Now, given a desired network lifetime and the active lifetime of individual sensor nodes, it is easy to compute the value of $p$, which also happens to be the expected duty cycle of each node. In addition, this approach of uniform duty cycle will balance the energy consumption in the network.

We note here that the idea of dividing time into periods and allowing nodes to sleep during some periods has been explored previously. A sentry-based power management scheme was proposed in [12], where an active node is called a sentry. However, the issue of how to select the sentries dynamically is not addressed there. A scheme for sentry selection was proposed in [9]. In this scheme, a node backs off for an interval inversely proportional to its remaining energy and then uses a communication radius equal to the sensing radius to inform the neighbors in its sensing range of its intention to become a sentry, if it has not heard such a message from a neighbor already. Although basing the back-off interval on the remaining energy is a good heuristic to balance the energy consumption in the network, it does not lead to the determination of the probability of sentry selection given a desired network lifetime, unlike the RIS scheme. In addition, the RIS scheme frees a node from the task of checking with its neighbors as to whether it should become a sentry or not.

Balancing power consumption is only a desirable criterion. The main goal is to guarantee full coverage with sentry nodes. One of the goals of the sentry selection scheme proposed in [9] is to guarantee 1 -coverage with the sentry nodes. However, if not enough sensors have been deployed, then the sentry selection scheme of [9] can not guarantee 1coverage. Similarly, for guaranteeing $k$-coverage with sentry nodes, algorithms proposed in $[11,18]$ can be used by the nodes to decide (by interacting with their neighbors) if it is necessary for them to be awake during a period to ensure $k$ coverage of every point. But again, if not enough nodes have been deployed, then there is no way that these algorithms can guarantee $k$-coverage. Therefore, the development of a sound method to compute the appropriate number of sensors nodes to be deployed so that every node needs to be active only $p$ fraction of the time and still guarantee $k$-coverage of every point in the region, is a fundamental problem currently facing the deployment of sensor networks for the intrusion detection application.

In this paper, we develop theories that can be used to answer questions such as these. In addition, our theorems apply to a variety of scenarios and platforms, other than the one mentioned above. We consider sleep to be a form of failure. ${ }^{2}$ The sleeping sensors can be considered as "failed," for the duration of their sleep. The added benefit of this model is that the sleep probability can be readily combined with the probability of other forms of failure in sensor networks such as failure to detect events of interest, or packet loss. For a more extensive list of failures prevalent in sensor networks deployed for intruder detection, classification and tracking, we refer the reader to [2]. The problem we address in this paper is that of developing the critical conditions relating the number of nodes deployed, their sensing radius, and their failure probability such that even in the case of failures (or sleeping, or both), each point in the region is almost always covered by at least $k$ sensors. We consider three kinds of deployments: a $\sqrt{n} \times \sqrt{n}$ grid, random uniform (for all $n$ points), and Poisson (with density $n$ ). Considering deterministic and random deployments in the same spirit allows us to make some interesting observations. For example, we find that the number of sensors needed in the grid deployment with RIS sleeping is of the same order as that in the random deployments with RIS sleeping, with a second order difference due to the boundary condition in the grid deployment.

We would like to briefly mention a few reasons for requiring $k$-coverage rather than just 1 -coverage in applications.

1. In the intruder detection application, for the purposes of classification, it is necessary to have every intruder detected by not just one sensor, but by at least $k$ sensors [2]; with the value of $k$ depending on the desired accuracy of classification. The sensing range of some sensors such as magnetometer depends on the type of intruder, e.g. 2-3 meters for armed personnel and 5-7 meters for cars. As a result, the number of magnetometers detecting a

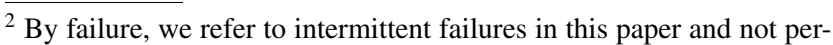
manent failures such as fail-stop.
} 
car is significantly higher than those detecting armed personnel. This fact is used in classifying a car from armed personnel [2].

2. A multi-hop wireless sensor network exhibits high loss of packets $[2,20]$, and so it is necessary to ensure that $k$ or more sensors detect an intruder to guarantee the reception of enough detection messages at the base station.

3. $k$ coverage is also needed to mask the false activation of individual sensors - differentiate the false activation of a sensor by wind or some other natural phenomenon from a real intrusion event. As the sensors in a large wireless sensor network are supposed to be cheap, false activation is likely to continue to occur in such networks.

4. Yet another reason for having $k$ sensors detect an event may be to improve the accuracy of tracking (e.g. $k$ coverage of a target improves the estimate of target location or its velocity by a factor of $\sqrt{k}$, if detection data are fused in an optimal manner [7]).

The rest of the paper is organized as follows: In Section 2, we describe our network model, summary of our contributions, application of our results, and some related work. In Section 3, we establish a result that we use in most of the proofs later on. In Section 4, we prove the phase transition conditions for 1 -coverage and $k$-coverage when the nodes are deployed in a regular grid. In this section, we also provide corrections to previously published results [16] on the grid deployment model. In Sections 5 and 6, we prove similar conditions when nodes are deployed randomly with uniform distribution and with Poisson distribution respectively. In Section 7, we present some results from simulation. Section 8 concludes the paper.

\section{Model, contributions, applications, and related work}

\subsection{The model and the problem definition}

We consider a square region of unit area where sensors are to be deployed according to the following models:

- $\sqrt{n} \times \sqrt{n}$ grid deployment of $n$ sensors, where each of the $n$ grid points hosts a sensor;

- random uniform deployment of $n$ sensors, where each of the $n$ sensors to be deployed has equal likelihood of falling at any location in the deployment area, independently of the other sensors.

- Poisson deployment with rate $n$, where sensors are deployed according to a 2-dimensional Poisson process. Notice that the number of sensors deployed in this model need not be exactly $n$.

We consider a particular point $t$ in time and assume that at time $t$ each of the deployed sensors is active with probability $p$, independently from the others, and inactive (due to either failure or sleeping) with probability $(1-p)$. We assume a disc-based sensing model where each active sensor has a sensing radius of $r$; any object within a disc of radius $r$ centered at an active sensor is reliably detected by it. A point in the region is said to be $k$-covered if it is within the sensing distance of $k$ or more active sensors. A region is said to be $k$-covered if every point in it is $k$-covered; and is said to be $n o t k$-covered if it contains some non- $k$-covered point(s).

The problems we address in this paper are the following:

1. What relation among $n, r, p$, and $k$ would be sufficient to guarantee that the probability of the entire region being $k$-covered approaches 1 as $n$ approaches infinity?

2. What relation among $n, r, p$, and $k$ would be sufficient to guarantee that the probability of the region being not $k$-covered approaches 1 as $n$ approaches infinity.

The conditions we seek as listed above are referred to as critical conditions for asymptotic coverage. The condition sought in 1 is a sufficient condition for asymptotic $k$-coverage. The second condition will be a sufficient condition for asymptotic non- $k$-coverage (while its negation will provide a necessary condition for asymptotic $k$-coverage). The gap between the two conditions, referred to as a window of phase transition, is a gray area where the behavior of asymptotic coverage is uncertain. In seeking critical conditions for asymptotic coverage, it is desired that the window of the phase transition converge to 0 as fast as possible; the faster the rate of convergence, the sharper the transition. The window of phase transition is also frequently used as an indicator of the expected behavior in finite cases.

\subsection{Notations and assumptions}

We adopt the following notations throughout the paper.

- $D_{r}(u)$ : the disc of radius $r$ centered at the point $u$.

- $\operatorname{Pr}[T]$ : the probability that event $T$ occurs.

- $\mathbb{E}[X]$ : the expected value of $X$.

- We say that an event $T(x)$ almost always occurs if $\lim _{x \rightarrow \infty} \operatorname{Pr}[T(x)]=1$. If it is clear which parameter goes to infinity, then we simply say that $\operatorname{Pr}[T]$ approaches 1 .

- We write $g(x)=o(f(x))$ iff $\lim _{x \rightarrow \infty} g(x) / f(x)=0$, $g(x)=\omega(f(x))$ iff $\lim _{x \rightarrow \infty} g(x) / f(x)=\infty$, and $g(x) \approx$ $f(x)$ iff $\lim _{x \rightarrow \infty} g(x) / f(x)=1$.

- We use $d(u, v)$ to denote the Euclidean distance between points $u$ and $v$.

- The parameters $r(n), r^{\prime}(n), p(n), c(n)$, and $c^{\prime}(n)$ are all functions of $n$. For ease of readability, we use $r, r^{\prime}, p, c$, and $c^{\prime}$ in their place, while still retaining the meaning that these are functions of $n$.

- For ease of presentation, we use $\sqrt{n}$ for $\lfloor\sqrt{n}\rfloor$ and $\sqrt{\ell}$ for $\lfloor\sqrt{\ell}\rfloor$. Since our calculations are robust, this does not affect our results. 
- We assume $\lim _{\sup } \rightarrow \infty p(n)<1$, unless stated otherwise.

We also assume that $n p \rightarrow \infty$ as $n \rightarrow \infty$.

- We assume $r(n) \rightarrow 0$ as $n \rightarrow \infty$.

\subsection{Contributions}

A function $\phi(n p)$ is slowly growing if it is monotonically increasing, goes to infinity as $n \rightarrow \infty$, and is $o(\log \log (n p))$. Throughout this paper, $\phi(n p)$ always denotes a slowly growing function. Let

$c(n)=\frac{n p \pi r^{2}}{\log (n p)}$.

In this paper, we make the following contributions on the issue of coverage of a square region of the unit area:

1. For the grid deployment, we prove that if there exists a slowly growing function $\phi(n p)$ such that $c(n) \geq 1+$ $[\phi(n p)(1+\sqrt{p \log (n p)})+k \log \log (n p)] / \log (n p)$, then all the points in the region are almost always $k$-covered as $n$ approaches infinity.

2. We also prove for the grid deployment that if $c(n) \leq 1$ $-[\phi(n p)(1+\sqrt{p \log (n p)})+\log \log (n p)] / \log (n p)$ for some (slowly growing function) $\phi(n p)$, then as $n$ approaches infinity there almost always exists a point in the region that is not 1 -covered. Certainly, if a point is not 1 -covered, then it is not $k$-covered, as well.

3 . For the random uniform deployment, we prove that if $c(n) \geq 1+[\phi(n p)+k \log \log (n p)] / \log (n p)$ for some $\phi(n p)$, then all the points in the region are almost always $k$ covered as $n$ approaches infinity.

4. We also prove for the random uniform deployment that if $c(n) \leq 1-[\phi(n p)+\log \log (n p)] / \log (n p)$ for some $\phi(n p)$, then as $n$ approaches infinity there almost always exists a point in the region that is not 1 -covered.

5. For Poisson distribution, we prove the same conditions as for the uniformly distributed nodes.

6. Finally, we provide corrections to previously published results. We show that a necessary condition derived in [16] (Proposition 2.1) does not hold. This also affects the claim made in [16] that asymptotic connectivity does not imply asymptotic coverage. This claim now remains open.

Remark 2.1. For the grid deployment, we have accounted for the boundary condition. In the case of random deployments, the number of sensors in the disc $D_{r}(u)$ for any point $u$ is independent of its location. However, in the grid deployment model, number of sensors in a disc $D_{r}(u)$ depends on the location of $u$. Therefore, for the grid deployment, we obtain a coarser estimate for the number of sensors in $D_{r}(u)$, which results in the extra term, $\phi(n p) \sqrt{p \log (n p)}$. This indicates that there is some difference between the deterministic and random models with respect to coverage.

Notice that both critical functions given by us for the case of $k$-coverage and non $k$-coverage converge to 1 . Therefore, for $k$-coverage, the critical value of $n p \pi r^{2} / \log (n p)$ is 1 . If we were to state our conditions as $\liminf c(n)>1$ and $\lim \sup c(n)>1$, then although they look simpler, they are not very useful for applications as they do not provide any information on the rate of convergence. On the other hand, our conditions provide a window of phase transition that provides a guidance on what behavior to expect in finite cases. For instance, for 1 -coverage in the random uniform deployment case, $2[\phi(n p)+\log \log (n p)] / \log (n p)$ is the window of phase transition for 1 -coverage that converges to 0 as $n \rightarrow \infty$, and at the same time provides useful guidance in finite cases. Figure 1 illustrates this situation for the probability of 1 -coverage. Simulation results in Section 7 illustrate this point further with actual results that exhibit a behavior similar to the one shown in Fig. 1. A more formal discussion on phase transition can be found in literature on percolation theory [14] and random graphs [4]

We note that although we are motivated mostly by the need to find out the number of sensors $n$ to be deployed given $p$, $r$, and $k$, to achieve $k$-coverage, our conditions allow the computation of any one parameter out of $n, p, r$, and $k$ given the other three, assuming the number of sensors is large.

Finally, we note that after the events are sensed, the notification needs to be sent to the base station. Consequently, the network must be connected. For the random uniform deployment, the conditions for $k$-connectivity has been derived in $[3,13]$ when $p=1$. These conditions can also be shown

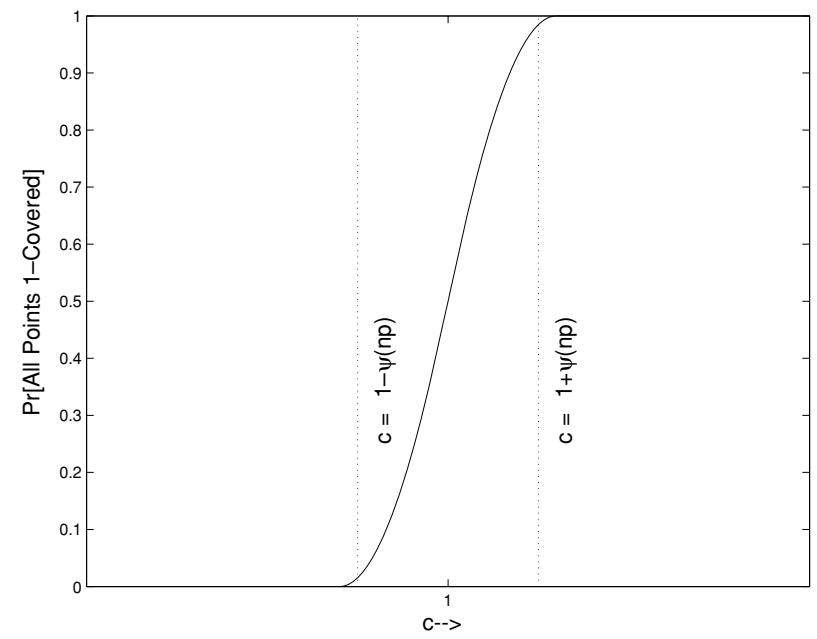

Fig. 1 An illustration of the phase transition window. Let $\psi(n p) \rightarrow$ 0 as $n p \rightarrow \infty$. When $c \leq 1-\psi(n p)$, our results predict that $\operatorname{Pr}$ [All points 1 -covered] should approach 0 and when $c \geq 1+\psi(n p)$, it should approach 1 . The gap between $1-\psi(n p)$ and $1+\psi(n p)$ is the window of phase transition; where the behavior of the probability is uncertain 
to hold for Poisson distribution. However, it is still an open issue whether similar conditions can be shown to hold for the $k$-connectivity in the case of grid deployment when the sensors are allowed to sleep according to the RIS scheme. A (not so sharp) sufficient condition for connectivity for all the three models of deployment considered here can be easily derived using an earlier published result [18] and our results on $k$ coverage. It has been shown earlier [18] that if $k$-coverage is guaranteed, then using a communication radius of twice the sensing radius ensures $k$-connectivity. Therefore, our sufficient conditions for $k$-coverage also give sufficient conditions for $k$-connectivity, although they are not as sharp as those for $k$-coverage.

\subsection{Applications of our results}

This work was motivated by user requirements of a real project. As such, our results are applicable to real projects. Our results can be used both in the initial deployment and in dynamic reconfiguration after the sensors have been deployed.

\subsubsection{Initial deployment}

- Determining the sensor density: Using our results for $k$ coverage, an appropriate value for the number of sensors can be computed, given the sensing radius $(r)$, desired lifetime of the network $(p)$, and the level of coverage desired $(k)$, in order to guarantee that all the points in the region are $k$-covered. Using an appropriate value for the level of coverage $(k)$ will also guarantee the classification of the intruders.

- Tolerating environmental losses: If at the time of deployment, the probability of missing an event by a sensor is known, then this probability can be multiplied with the sleep probability to give a more accurate value of $p$ to be used in the calculations, which, in turn, will allow for tolerance to event detection failures. Similarly, if other loss behaviors are known to be independently and uniformly distributed, their probability can be subsumed in $p$ to get more accurate calculations in real life.

\subsubsection{Dynamic reconfiguration}

Here, we assume that a network reprogramming service exists to communicate the values of parameters from the base station to all the sensors, as is the case with sensor networks being built today [2].

- Changing the level of coverage: The value of $k$ in $k$ coverage can be changed dynamically by choosing a different value of $k$ when the sensors have already been deployed. This can be done by distributing a new value of $p$ to the network corresponding to the new value of $k$. If it is desired to have different levels of coverage at different times of the day, then precomputed values of $p$ can be provided to the sensors in a one-time communication or by having these values programmed in them before the deployment.

- Increasing the desired lifetime: Consider a scenario where multiple sensors are mounted on the nodes [10] (which is usually the case $[2,17])$ and it is discovered after the deployment that the network is required to last longer than was originally planned. Since different sensors have different sensing radius, one way to solve this problem may be to use only the longer range sensors for full $k$ coverage. In such a case, a new value of $p$ (a lower one) can be distributed to the network corresponding to the new value of $r$. The nodes can now start sleeping with a higher probability; making the network last longer.

- Dealing with failures at the time of deployment: Consider a scenario where it is determined after the deployment that $50 \%$ of the sensors have failed due to errors in the deployment process. If the original value of $p$ was at most 0.5 , then it is possible to compute a new value of $p$ using the reduced value of $n$, in order to achieve the same level of coverage. This new value of $p$ can be communicated to the network and the network can function as was originally planned except that the network will now have a reduced lifetime. Additional sensors can be deployed at a later date in order to compensate for this loss.

- Increasing the probability of coverage: After the network has been deployed, the $k$-coverage algorithms $[11,18]$ can be run on the nodes to improve the probability of coverage.

Similar types of dynamic reconfiguration can be performed by dynamically changing the value of $p$. In some types of sensors, it may be possible to dynamically change the value of $r$ too.

\subsection{Related work}

The work closest to ours is [16], where necessary and sufficient conditions for 1 -coverage and 1 -connectivity are derived when $n$ sensors are deployed in a $\sqrt{n} \times \sqrt{n}$ grid and each sensor is allowed to fail independently with probability $(1-p)$. It is claimed in [16] that $\lim \inf n p r^{2} / \log (n) \geq 1 / \pi$ is a necessary condition for 1 -coverage (Proposition 2.1 in [16]), which, as to be discussed later in Section 4.3, however, is not true.

It is also claimed in [16] that $\lim \inf n p r^{2} / \log (n)>4 / \pi$ is a sufficient condition for 1 -coverage, which in our notation can be written as

$c(n)>\frac{4 \log (n)}{\log (n p)}$ 
where $c(n)$ is as defined in (1). This condition has a stronger requirement than our sufficient condition for 1 -coverage due to the $\log (n) / \log (n p)$ term in the numerator instead of $\log (n p)$. Another difference between our work and [16] is that we derive conditions for $k$-coverage, whereas the work in [16] focused on 1 -coverage. Also, we use different techniques to derive our conditions than the ones used in [16].

Poisson distribution has been studied extensively in the literature. In [8], the problem of 1 -coverage has been addressed when nodes are always active (i.e. $p=1$ in our model). It is proved (Theorem 3.11 in [8]) that the probability of non-coverage is less than $\min \left(1,3\left(1+\pi r^{2} n^{2}\right) e^{-\pi r^{2} n}\right)$. As was shown in [6], this results in a sufficient condition for 1-coverage:

$\frac{n \pi r^{2}}{\log n}=1+\frac{\phi(n)+\log \log (n)}{\log (n)}$.

This condition is a special case of our Theorem 6.1 with $k=1$.

In the same theorem in [8] (as was shown in [6]), it is also proved that if $n \pi r^{2} / \log n \leq 1-[\phi(n)-\log \log (n)] /$ $\log (n)$, then the probability of non-coverage is greater than $1 / 20$. Our sufficient condition for non-coverage (Theorem 6.2) is slightly stronger than this, but our condition guarantees non-coverage with probability tending to 1 .

In [19], sensors are deployed according to a Poisson distribution with rate $\lambda$ in a square region with side length $\ell$. Each sensing disk is assumed to have unit area. It is proved that the square is almost always $k$-covered if $\lambda$ satisfies

$\lambda=\log \left(\ell^{2}\right)+(k+1) \log \log \left(\ell^{2}\right)+c(\ell)$

and $c(\ell) \rightarrow \infty$. It is also proved that the square is almost always non- $k$-covered if $\lambda$ satisfies (3) and $c(\ell) \rightarrow-\infty$. Although our condition (61) may appear to be better than the sufficient condition of [19] and appear contradictory to the sufficient condition for non $k$-coverage of [19], our sufficient condition for $k$-coverage is the same as the one derived in [19] (after careful scaling). We provide the details of how to transform the results derived under the model used in [19] to our model of unit square in Section 6, and vice versa. Since we have shown our result to hold for the regions close to the boundary (which requires a more difficult proof than for regions away from the boundary), it shows that the results of [19] also hold for regions close to the boundary.

The sufficient condition for non- $k$-coverage derived in [19] for Poisson distributed nodes is actually stronger than our condition (62). We conjecture that a similar condition for non- $k$-coverage will hold for uniform and grid deployment.
We are not aware of any work on the issue of $k$-coverage in the case of random uniform and grid deployment, when nodes are allowed to fail (and/or sleep).

\section{Virtual grid}

We start our exploration by showing that if a certain (finite) set of points in the unit square is $k$-covered by a sensor network with a certain sensing radius, then the entire region is $k$-covered by the same sensor network with a slightly larger sensing radius. The set of points we will use, denoted by $L$, is the set of all grid-points of a $\sqrt{\ell} \times \sqrt{\ell}$ virtual grid on the unit square region as illustrated in Fig. 2. The $L$ in the next lemma refers to this set. With this result, whenever wishing to show the unit square $k$-covered, we will only need to show that $L$, with an appropriate value of $\ell$, is $k$-covered.

Lemma 3.1. Let $r, r^{\prime}$ and $\ell$ be constants such that $r^{\prime}=r-$ $1 / \sqrt{2 \ell}$, and let $L$ be the set of all grid points of the $\sqrt{\ell} \times \sqrt{\ell}$ virtual grid. If $L$ is $k$-covered by a network with sensing radius $r^{\prime}$, then the entire unit square region is $k$-covered by the same network but with sensing radius $r$.

Proof: Let $v$ be an arbitrary point in the square region. Without loss of generality, we may assume it is inside the square formed by some set of four points $a, b, c$, and $d$ on the virtual grid as shown in Fig. 3. Also, without loss of generality, we may assume that it is closest to the point $a$. By assumption, there exist at least $k$ active sensors that cover point $a$. Let one of these be located at point $u$ as shown in Fig. 3. Then, $d(u, a)<r^{\prime}$. From triangle inequality,

$d(u, v) \leq d(u, a)+d(a, v)<r^{\prime}+\frac{1}{\sqrt{2 \ell}}=r$.

The same holds for the other active sensors covering point $a$. Therefore, we conclude that every point in the region is

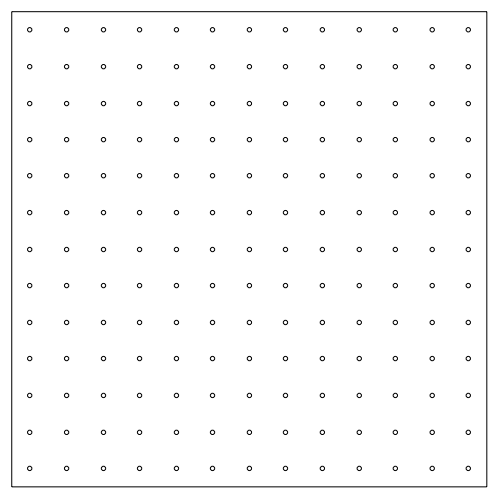

Fig. 2 The unit square region tiled with a virtual grid with $\ell=169$ points 


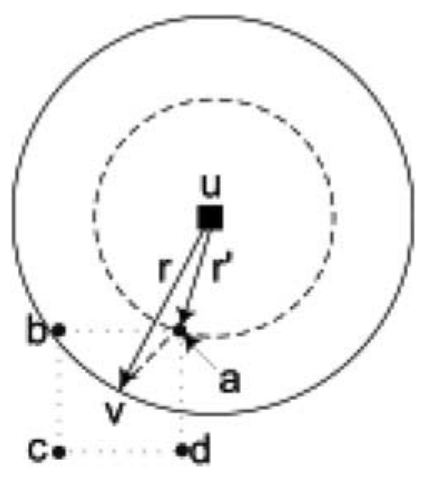

Fig. 3 A set of four nearest points on the virtual grid

$k$-covered by using a sensing radius of $r$, if all the points on the virtual grid are $k$-covered using a sensing radius of $r^{\prime}$.

Remark 3.1. If we use the points of a triangular lattice instead of the grid-points of a square grid for the virtual points that are to be covered, then the value of $r^{\prime}$ only needs to be $(r-\sqrt{2 /(3 \sqrt{3} \ell)})$. This is the optimal topology for the virtual points and this value of $r^{\prime}$ should be used in practice to compute the required number of nodes to achieve coverage. As it does not give a significant improvement on the window, we stick with the grid layout.

\section{Grid deployment}

Suppose $n$ sensors are deployed in a unit square region in a $\sqrt{n} \times \sqrt{n}$ grid fashion as shown in Fig. 4, with each grid point hosting a sensor. The $\sqrt{n} \times \sqrt{n}$ sensor grid is different from the $\sqrt{\ell} \times \sqrt{\ell}$ virtual grid introduced in Section 3. The former refers to a set of sensors, whereas the latter is used to define a set of points, $L$, in the region.

We are interested in asymptotic $k$-coverage of the unit square. For ease of exposition, we first develop the conditions

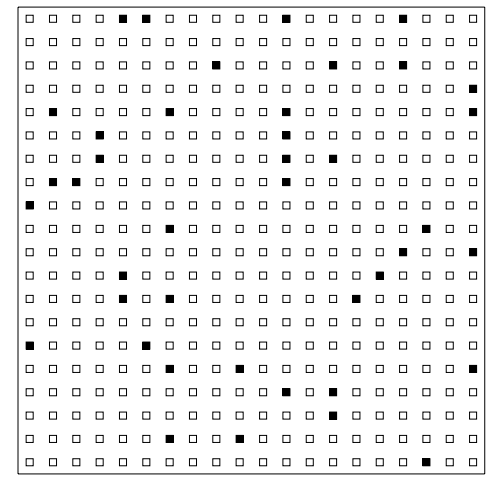

Fig. 4 Grid deployment of sensors on a unit square with $n=400$ and $p=0.1$. The filled squares represent active sensors for 1 -coverage in Section 4.1; and then extend the results to $k$-coverage in Section 4.2.

\subsection{1-Coverage conditions}

Assume that each sensor has a sensing radius of $r$, and at any particular point of time $t$, it remains active with probability $p$ and either fails or sleeps with probability $(1-p)$.

Let $\phi(n p)$ and $\phi^{\prime}(n p)$ be any two slowly growing functions such that $\phi(n p)=\Theta\left(\phi^{\prime}(n p)\right)$. (Here, we use the standard $\Theta$ notation as in complexity theory.) Let $L$ be the set of virtual grid points as defined in Section 3, with $\ell=(n p) \phi^{\prime}(n p) \log (n p)$. The following lemma indicates a sufficient condition for $L$ to be almost always covered.

Lemma 4.1. If, for some slowly growing $\phi(n p), p$ and $r$ satisfy

$c(n)=1+\frac{\phi(n p)(1+\sqrt{p \log (n p)})+\log \log (n p)}{\log (n p)}$

for sufficiently large $n$, then $L$ is almost always covered.

Proof: Partition $L$ into three sets: $I, C$, and $S$. Set $I$ contains all the inner (grid) points, which are at least $r$ distance away from every side. Set $C$ contains the points which are less than $r$ distance away from a pair of neighboring sides (close to a corner point). Finally, let $S$ contain the rest of the points in $L$ (each close to a side). $L$ is almost always covered iff $I, C$ and $S$ are each almost always covered.

For each point $u \in L$, let $A(u)$ denote the event that $u$ is covered; and $\overline{A(u)}$, its negation. Then, for $Z=C, I$, or $S$,

$$
\operatorname{Pr}[Z \text { is covered }]=\operatorname{Pr}\left[\bigwedge_{u \in Z} A(u)\right] .
$$

To show that $\operatorname{Pr}[Z$ is covered $] \rightarrow 1$, we make use of Janson's Inequality to get a lower bound on $\operatorname{Pr}\left[\wedge_{u \in Z} A(u)\right]$. Janson's Inequality [1, Theorem 8.1.1] states that if events $\overline{A(u)}$ for $u \in Z$ satisfy

$\operatorname{Pr}\left[\overline{A(u)} \mid \wedge_{v \in V} A(v)\right] \leq \operatorname{Pr}[\overline{A(u)}]$

for all index sets $V \subset Z, u \notin V$, then

$\operatorname{Pr}\left[\bigwedge_{u \in Z} A(u)\right] \geq \prod_{u \in Z} \operatorname{Pr}[A(u)]$

The condition in (5) is satisfied in our case, since the probability that a point on the virtual grid is not covered, given the information that its neighboring points on the virtual grid are covered, is smaller than its unconditional probability of not being covered. This is because an active sensor covers 
multiple points on the virtual grid. Therefore, Janson's Inequality holds here.

Now, $\operatorname{Pr}[Z$ is covered $] \rightarrow 1$ if $\prod_{u \in Z} \operatorname{Pr}[A(u)] \rightarrow 1$, which we establish in the following by considering $I, C$, and $S$ separately. We note that the proof for the three sub-regions are different and the proof for the sub-region $S$ is the most challenging one.

Case 1. First consider $I$, noting that $|I| \leq \ell$. For points $u \in$ $I$, event $\overline{A(u)}$ occurs iff all the sensors in disc $D_{r}(u)$ are inactive. The number of sensors that are within disc $D_{r}(u)$ is $m \geq m_{l}=\pi r^{2} n-2 \pi r \sqrt{n}$. Thus,

$\operatorname{Pr}[\overline{A(u)}]=(1-p)^{m} \leq(1-p)^{m_{l}} \leq e^{-p m_{l}}$.

It can be easily verified that $p m_{l}=\alpha \log (n p)$, where

$\alpha=c-\sqrt{4 p \pi c / \log (n p)}$.

Therefore,

$\operatorname{Pr}[\overline{A(u)}] \leq e^{-p m_{l}}=(n p)^{-\alpha}$

and

$\operatorname{Pr}[A(u)] \geq 1-(n p)^{-\alpha}$.

Using Janson's Inequality (6), the probability that all points in $I$ are covered is given by

$$
\begin{aligned}
\operatorname{Pr}[I \text { is covered }] & \geq \prod_{i \in I} \operatorname{Pr}[A(i)] \\
& \geq\left(1-(n p)^{-\alpha}\right)^{\ell} \\
& \geq \exp \left(-5 \ell(n p)^{-\alpha}\right) .
\end{aligned}
$$

Here we have used the fact that $(1-x) \geq e^{-5 x}$ if $x \leq 0.99$. Since $\alpha \rightarrow 1$ and $n p \rightarrow \infty,(n p)^{-\alpha} \leq 0.99$ for sufficiently large $n$. The right hand side of (11) goes to 1 if the exponent of $e,-5 \ell(n p)^{-\alpha}$, goes to 0 or, equivalently, if $\log \left(\ell(n p)^{-\alpha}\right)$ goes to $-\infty$, which we show below. Using (8), (4) and the definition $\ell=(n p) \phi^{\prime}(n p) \log (n p)$, we have

$$
\begin{aligned}
\log \left(\ell(n p)^{-\alpha}\right)= & -\phi(n p)-\phi(n p) \sqrt{p \log (n p)} \\
& +\log \left(\phi^{\prime}(n p)\right)+\sqrt{4 \pi c} \sqrt{p \log (n p)} .
\end{aligned}
$$

The first and the second terms in the right hand side of the equation dominate the third and the fourth, respectively. Therefore, $\log \left(\ell(n p)^{-\alpha}\right) \rightarrow-\infty$, and $\operatorname{Pr}[I$ is covered $] \rightarrow 1$.

Case 2. Next, consider $C$. For $u \in C$, the number of sensors that are within disc $D_{r}(u)$ is at least $m_{l} / 4$, where $m_{l}$ is as defined in Case 1. A similar argument as for (10) results in

$\operatorname{Pr}[A(u)] \geq 1-(n p)^{-\alpha / 4}$.

Note that $|C| \leq 4 r^{2} \ell\left(1+\frac{1}{r \sqrt{\ell}}\right)^{2} \leq 8 r^{2} \ell$. Proceeding as in (11) (again using Janson's Inequality) yields

$\operatorname{Pr}[C$ is covered $] \geq \exp \left(-40 r^{2} \ell(n p)^{-\alpha / 4}\right)$

$$
\geq \exp \left(-40 e^{\log \left(r^{2} \ell(n p)^{-\alpha / 4}\right)}\right) \text {. }
$$

The inner exponent, $\log \left(r^{2} \ell(n p)^{-\alpha / 4}\right)$, can be written as follows (using (8) and $r^{2} \ell=c \log ^{2}(n p) \phi^{\prime}(n p) / \pi$ ):

$$
\log \left(\frac{c \log ^{2}(n p) \phi^{\prime}(n p)}{\pi}\right)-\frac{c \log (n p)}{4}+\sqrt{\frac{p \pi c \log (n p)}{4}} .
$$

The whole expression goes to $-\infty$, forcing the right hand side of (13) to approach 1.

Case 3. Finally, consider $S$. We tried to use the inequality $\operatorname{Pr}[A(u)] \geq 1-(n p)^{-\alpha / 2}$ (cf. (10) and (12)), but as it turned out, we needed a tighter bound to obtain the desired result.

Let $S_{l}$ be the set of points in $S$ that are close to the unit square's left side. $S$ is almost always covered if $S_{l}$ is almost always covered. Label the columns of grid points in $S_{l}$ from 0 to $f-1$, starting with the leftmost column, where $f$ is the number of columns in $S_{l}$. For a grid point $u$ in column $j$, the area of the disc $D_{r}(u)$ that is within the square region is more than $\pi r^{2} / 2+j r / \sqrt{\ell}$ and the number of sensors is at least $m_{j}=\pi r^{2} n / 2+j r n / \sqrt{\ell}$. Therefore, for a point $u \in S_{l}$ in column $j$,

$\operatorname{Pr}[\overline{A(u)}] \leq(1-p)^{m_{j}} \leq e^{-p m_{j}}=(n p)^{-\sigma_{j}}$,

where $\sigma_{j}=c / 2+j c /(\pi r \sqrt{\ell})$. From (14), the fact $(1-$ $x) \geq e^{-5 x}$ if $x \leq 0.99$, and by observing that $(n p)^{-\sigma} \leq 0.99$ for sufficiently large $n$, it follows that

$$
\operatorname{Pr}[A(u)] \geq 1-(n p)^{-\sigma_{j}} \geq \exp \left(-5(n p)^{\sigma_{j}}\right) .
$$

There are no more than $\sqrt{\ell}$ grid points in a column. By Janson's Inequality (6), the probability that all the points in $S_{l}$ are covered is given by

$$
\begin{aligned}
\operatorname{Pr}\left[\bigwedge_{u \in S_{l}} A(u)\right] & \geq \prod_{j=0}^{f-1}\left(\exp \left(-5(n p)^{\sigma_{j}}\right)\right)^{\sqrt{\ell}} \\
& \geq \exp \left(-5 \sqrt{\ell}(n p)^{-c / 2} \sum_{j=0}^{f-1} w^{j}\right)
\end{aligned}
$$


where

$$
w=(n p)^{-c /(\pi r \sqrt{\ell})}=\exp \left(-\sqrt{\frac{c}{\pi \phi(n p)}}\right) .
$$

We have used the relation $r \sqrt{\ell}=\log (n p) \sqrt{c \phi^{\prime}(n p) / \pi}$ in obtaining the last equality.

The right hand side of (15) approaches 1 if the exponent of $e$ goes to 0 , which we show in the following. We first note that since $\sum_{j=0}^{f-1} w^{j}$ is the sum of a geometric series and $f \rightarrow \infty$, therefore $(1-w)^{-1}$ is a good approximation for this summation. Using this value, the exponent of $e$ in (15) can be expressed as

$$
\frac{-5 \exp ((-\phi(n p)-\phi(n p) \sqrt{p \log (n p)}+\log (\phi(n p))) / 2)}{1-\exp \left(-\sqrt{\frac{c}{\pi \phi(n p)}}\right)},
$$

which can be easily shown to converge to 0 using L'Hospital's Rule. Consequently, the right hand side of (15) approaches 1. We note that the rate of convergence is the slowest for this case.

Remark 4.1. Since the convergence of the bound is the slowest for the points close to the sides, it is possible to save some nodes in the deployment by maintaining a higher density of nodes on the sides and a lower density in the inner region.

Now, we show that the same condition, (4), ensures asymptotic coverage of the unit square.

Theorem 4.1. If, for some slowly growing function $\phi(n p)$, $p$ and $r$ satisfy

$c(n)=1+\frac{\phi(n p)(1+\sqrt{p \log (n p)})+\log \log (n p)}{\log (n p)}$

for sufficiently large $n$, then the entire unit square region is almost always covered.

Proof: Let $L$ be the set of virtual grid points introduced in Section 3, with $\ell=(n p) \phi(n p) \log (n p)$; let $r^{\prime}=r-1 / \sqrt{2 \ell}$; and let $c^{\prime}(n)=n p \pi r^{\prime 2} / \log (n p)$. We will show

$c^{\prime}(n)=1+\frac{\phi^{\prime}(n p)(1+\sqrt{p \log (n p)})+\log \log (n p)}{\log (n p)}$

for some slowly growing function $\phi^{\prime}(n p)=\Theta(\phi(n p))$. Once this is proved, then by Lemma $4.1, L$ is almost always covered; and then by Lemma 3.1, the unit square region is almost always covered.
To establish (17), we obtain the following using the definitions of $c^{\prime}, c, r^{\prime}$ and $\ell$ :

$$
\begin{aligned}
c^{\prime}(n) & =c(n)\left(1-\frac{2}{\sqrt{2 r^{2} \ell}}+\frac{1}{2 r^{2} \ell}\right) \\
& =c(n)\left(1-o\left(\frac{1}{\log (n p)}\right)\right) \\
& =c(n)-\frac{o(c(n))}{\log (n p)}
\end{aligned}
$$

Substituting the equality from (16) in (18) results in

$c^{\prime}(n)=1+\frac{\phi(n p)(1+\sqrt{p \log (n p)})-o(c(n))+\log \log (n p)}{\log (n p)}$

which is equivalent to

$c^{\prime}(n)=1+\frac{\phi^{\prime}(n p)(1+\sqrt{p \log (n p)})+\log \log (n p)}{\log (n p)}$

where

$\phi^{\prime}(n p)=\phi(n p)-o(c(n))$.

This establishes (17) and thereby proves the theorem.

Corollary 4.1. If, for some slowly growing $\phi(n p), p$ and $r$ satisfy

$c(n) \geq 1+\frac{\phi(n p)(1+\sqrt{p \log (n p)})+\log \log (n p)}{\log (n p)}$

for sufficiently large $n$, then the entire unit square region is almost always covered.

Proof: If $c(n)$ satisfies (20), there exists an $r_{l} \leq r$ for which $c_{l}(n)=n p \pi r_{l}^{2} / \log (n p)$ satisfies (16). The region is almost always covered when $r_{l}$ is used, and remains so when a larger sensing radius $r$ is used.

Corollary 4.2. A simple sufficient condition for asymptotic coverage of the unit square region is $\lim _{n \rightarrow \infty} c(n)>1$.

Proof: The right hand side of (17) approaches 1 as $n$ goes to infinity. Thus, if $\lim _{n \rightarrow \infty} c(n)>1$, then for any slowly growing function $\phi(n p),(17)$ holds for sufficiently large $n$.

We now prove a sufficient condition for asymptotic noncoverage of the unit square when a sensing radius of $r$ is used. Such a result is stronger than a mere necessary condi- 
tion for (asymptotic) 1 -coverage. In the next theorem, $c(n)$ refers to the same function as defined in (1).

Theorem 4.2. Assume $\lim _{n \rightarrow \infty} p=0$. If, for some slowly growing function $\phi(n p), p$ and $r$ satisfy

$c(n)=1-\frac{\phi(n p)(1+\sqrt{p \log (n p)})+\log \log (n p)}{\log (n p)}$

for sufficiently large $n$, then the unit square region is almost always not 1-covered.

Proof: It suffices to show $I$, the set of inner grid points as defined in the proof of Lemma 4.1, to be almost always not 1 covered. (Recall the dimensions of the virtual grid: $\sqrt{\ell} \times \sqrt{\ell}$, where $\ell=n p \phi(n p) \log (n p)$.)

For any point $i \in I$, let $X_{i}$ be the indicator random variable of event $\overline{A(i)}$, i.e. $X_{i}=1$ if $i$ is not $l$-covered and 0 , otherwise. Let $X$ be the number of points in $I$ which are not 1 covered. Then, $X=X_{1}+X_{2}+\cdots+X_{\ell}$. I is almost always not 1 -covered iff almost always $X>0$. To show that almost always $X>0$, we use Corollary 4.3.4 of [1], which states that almost always $X>0$ if $\mathbb{E}[X] \rightarrow \infty$ and $\Delta=o\left(\mathbb{E}^{2}[X]\right)$, where

$\Delta=\sum_{u \sim v} \operatorname{Pr}[\overline{A(u)} \wedge \overline{A(v)}]$

Here, $u \sim v$ means that $u \neq v$ and $\overline{A(u)}$ and $\overline{A(v)}$ are not independent.

First, we show $\mathbb{E}[X] \rightarrow \infty$. The event $\overline{A_{u}}$ occurs iff all the sensors in the disc $D_{r}(u)$ are inactive. The number of sensors $m$ that are within disc $D_{r}(u)$ is at most $m_{u}=\pi r^{2} n$. Using the relation $c=n p \pi r^{2} / \log (n p)$, we obtain

$\operatorname{Pr}[\overline{A(u)}]=(1-p)^{m} \geq(1-p)^{m_{u}} \approx e^{-p m_{u}}=(n p)^{-c}$.

Here, we have used the fact that $(1-x)^{y} \approx e^{-y x}$ if $y \rightarrow \infty$ and $x \rightarrow 0$. Now, we compute the expected number of noncovered points on the virtual grid, $\mathbb{E}[X]$.

$\mathbb{E}[X]=\ell \cdot \operatorname{Pr}[\overline{A(u)}] \geq \ell(n p)^{-c}=\exp \left(\log \left(\ell(n p)^{-c}\right)\right)$.

Using $\ell=(n p) \phi(n p) \log (n p)$, the assumption on $c$ from (21), and observing from (22) that $(n p)^{-c}=$ $\exp \left(-n p \pi r^{2}\right)$, the exponent of $e$ in (23) can be rewritten as follows:

$$
\begin{aligned}
\log \left(\ell(n p)^{-c}\right)= & \phi(n p)(1+\sqrt{p \log (n p)}) \\
& +\log (\phi(n p))+2 \log \log (n p) .
\end{aligned}
$$

The whole expression on the right hand side of (24) goes to $\infty$ forcing $\mathbb{E}[X]$ to go to $\infty$.

Next, we show $\Delta=o\left(\mathbb{E}^{2}[X]\right)$. For any two grid points $u$ and $v, u \sim v$ if the distance between $u$ and $v$ is less than $2 r$. For a given point $u$ on the virtual grid, there are two cases for points $v$ such that $u \sim v$ : (1) $0<d(u, v)<r$ and (2) $r \leq$ $d(u, v)<2 r$. We compute $\operatorname{Pr}[\overline{A(u)} \wedge \overline{A(v)}]$, conditioned on the above two disjoint cases.

Case 1: $0<d(u, v)<r$. It is obvious that $\operatorname{Pr}[\overline{A(u)} \wedge$ $\overline{A(v)}] \leq \operatorname{Pr}[\overline{A(u)}]$, and thus from (8) and (9) we have

$$
P_{r}=\operatorname{Pr}[\overline{A(u)} \wedge \overline{A(v)} \mid 0<d(u, v)<r] \leq(n p)^{-\alpha} .
$$

Case 2: $r \leq d(u, v)<2 r$. The area of $D_{r}(u) \cap D_{r}(v)$ is less than $2 \pi r^{2} / 3-\sqrt{3} r^{2} / 2$; hence the area of $D_{r}(u) \cup D_{r}(v)$ is more than $4 \pi r^{2} / 3+\sqrt{3} r^{2} / 2$, and the number of sensors in $D_{r}(u) \cup D_{r}(v)$ is more than $m_{2 r}=4 \pi r^{2} n / 3$ when $r \sqrt{n}>$ 11.67, which trivially holds since $r \sqrt{n} \rightarrow \infty$. Similarly as in (7), but using the definition of $m_{2 r}$ from above, we obtain

$$
\begin{aligned}
P_{2 r} & \leq \operatorname{Pr}[\overline{A(u)} \wedge \overline{A(v)} \mid r<d(u, v)<2 r] \\
& \leq(1-p)^{m_{2 r}} \leq e^{-p m_{2 r}}=(n p)^{-4 c / 3} .
\end{aligned}
$$

For a given point $u$, there are $\ell_{r} \approx \pi r^{2} \ell$ points $v$ such that $0<d(u, v)<r$ and there are $\ell_{2 r} \approx 3 \pi r^{2} \ell$ points $v$ such that $r \leq d(u, v)<2 r$. There are no more than $\ell$ choices for selecting the point $u$. However, because of double counting, since $u \sim v$ iff $v \sim u$, we have to divide it with 2. Using (25) and (26), we obtain

$$
\begin{aligned}
\Delta & \leq \frac{\ell}{2}\left(\ell_{r} P_{r}+\ell_{2 r} P_{2 r}\right) \\
& \leq \frac{\ell}{2}\left(\ell_{r}(n p)^{-\alpha}+\ell_{2 r}(n p)^{-4 c / 3}\right) .
\end{aligned}
$$

From (23) and (27), it follows

$$
\frac{\Delta}{\mathbb{E}[X]^{2}} \leq \frac{\ell_{r}(n p)^{-\alpha}+\ell_{2 r}(n p)^{-4 c / 3}}{2 \ell(n p)^{-2 c}} .
$$

The $\ell_{r}(n p)^{-\alpha}$ term dominates the $\ell_{2 r}(n p)^{-4 c / 3}$ term in (28). Therefore, showing that $\ell_{r}(n p)^{-\alpha} /\left(2 \ell(n p)^{-2 c}\right)$ approaches 0 , is enough to show that the whole expression on the right hand side of (28) approaches 0 as $n \rightarrow \infty$. Using the relation $\ell_{r} \leq \log (n p) \ell /(n p)$, which follows since $c \leq 1$, we obtain

$\frac{\ell_{r}(n p)^{-\alpha}}{2 \ell(n p)^{-2 c}} \leq \log (n p)(n p)^{2 c-\alpha-1}$ 
Taking the logarithm of the right hand side of (29) and manipulating it using (8) and (21), we obtain

$$
\begin{aligned}
& \log \left(\log (n p)(n p)^{c-\alpha}(n p)^{c-1}\right) \\
& =-\phi(n p)-\phi(n p) \sqrt{p \log (n p)}+\sqrt{4 p \pi c \log (n p)} .
\end{aligned}
$$

The whole expression in the right hand side of (30) goes to $-\infty$ as $n \rightarrow \infty$, thereby forcing the right hand side of (29) (and hence that of (28)) to approach 0 . This proves $\Delta=$ $o\left(\mathbb{E}^{2}[X]\right)$.

Now that $\mathbb{E}[X] \rightarrow \infty$ and $\Delta=o\left(\mathbb{E}^{2}[X]\right)$, we conclude by Corollary 4.3.4 of [1] that $X>0$ almost always. In other words, almost always there exists a non-covered point in the region.

Corollary 4.3. Let $\lim _{n \rightarrow \infty} p=0$. If, for some slowly growing $\phi(n p), p$ and $r$ satisfy

$c(n) \leq 1-\frac{\phi(n p)(1+\sqrt{p \log (n p)})+\log \log (n p)}{\log (n p)}$

for sufficiently large $n$, then the unit square region is almost always not 1-covered.

Proof: When $c(n)$ satisfies (31), there exists an $r_{u} \geq r$ for which $c_{u}(n)=n p \pi r_{u}^{2} / \log (n p)$ satisfies (21), and so the region is almost always not 1 -covered using $r_{u}$. This implies that the region is automatically not 1 -covered when a smaller sensing radius of $r$ is used.

Corollary 4.4. If $\lim _{n \rightarrow \infty} p=0$, a simple necessary condition for asymptotic coverage is $\lim _{n \rightarrow \infty} c(n) \geq 1$.

Proof: Otherwise, if $\lim c(n)<1$, then (31) would hold (for any slowly growing $\phi(n p))$ and the unit square region would be almost always not 1 -covered.

\section{$4.2 k$-Coverage conditions}

As a generalization to Theorem 4.1, we derive in this section a sufficient condition for asymptotic $k$-coverage. We do not need a generalization to Theorem 4.2 since a sufficient condition for non- 1 -coverage is also a sufficient condition for non$k$-coverage. As before, $\phi(n p)$ denotes a slowly growing function, $\ell=(n p) \phi(n p) \log (n p)$, and $c(n)=n p \pi r^{2} / \log (n p)$. from 1 .

Theorem 4.3. If, for some slowly growing function $\phi(n p)$, $p$ and $r$ satisfy

$c(n)=1+\frac{\phi(n p)(1+\sqrt{p \log (n p)})+k \log \log (n p)}{\log (n p)}$ for sufficiently large $n$, then the entire unit square region is almost always $k$-covered.

Proof: The proof is similar to that of Theorem 4.1. Thus, along the same line of reasoning as in that proof, we will show the following:

Claim 1: If $r$ and $p$ satisfy (32), then $r^{\prime}=r-1 / \sqrt{2 \ell}$ and $p$ satisfy

$c^{\prime}(n)=1+\frac{\phi^{\prime}(n p)(1+\sqrt{p \log (n p)})+k \log \log (n p)}{\log (n p)}$,

where $c^{\prime}(n)=n p \pi r^{\prime 2} / \log (n p)$ and $\phi^{\prime}(n p)$ is a slowly growing function such that $\phi^{\prime}(n p)=\Theta(\phi(n p))$.

Claim 2: If $r^{\prime}$ and $p$ satisfy (33), then $L$ is $k$-covered using the reduced sensing radius $r^{\prime}$, where $L$ is the set of grid points of the $\sqrt{\ell} \times \sqrt{\ell}$ virtual grid on the unit square region.

Once these two claims are proved, the theorem will immediately follow from Lemma 3.1.

To see Claim 1, we note that in the proof of Theorem 4.1, the arguments leading to (18) only uses the definition of $c$ from (1) and so it applies in the $k$-coverage case too. Substituting the equality from (32) in (18) yields (33) immediately.

To prove Claim 2, we follow the proof of Lemma 4.1 except that now we use $\overline{A_{k}(u)}$ in place of $\overline{A(u)}$, where $\overline{A_{k}(u)}$ is the event that a point $u$ on the virtual grid is not $k$-covered. We consider here only the case of $I$. The proof for sets $C$ and $S$ can be done with similar modifications as that presented here for $I$. Also, for simplicity, we write $r^{\prime}, c^{\prime}$ and $\phi^{\prime}(n p)$ as $r, c$ and $\phi(n p)$, respectively. With $\phi(n p)$ now denoting $\phi^{\prime}(n p)$, we will write the original $\phi(n p)$ which appears in the definition of $\ell$ as $\hat{\phi}(n p)$. Thus, $\ell=n p \hat{\phi}(n p) \log (n p)$. Note: $\phi(n p)=\Theta(\hat{\phi}(n p))$, from Claim 1 .

Let $\delta>0$ be any constant such that $\lim _{\sup _{n \rightarrow \infty}} p<1-$ $\delta$; such a constant exists since by assumption (Section 2.2), $\lim \sup _{n \rightarrow \infty} p<1$. Let $m$ be the number of sensors within disc $D_{r}(u)$; then $m \leq \pi r^{2} n$.

Let $N_{r}(u)$ denote the number of active sensors in $D_{r}(u)$, and let $P_{i}(u)=\operatorname{Pr}\left[N_{r}(u)=i\right]$. From (9), we have

$P_{0}(u)=(1-p)^{m} \leq(n p)^{-\alpha}$,

where, as defined in (8), $\alpha=c-\sqrt{4 p \pi c / \log (n p)}$.

For $i \geq 1$, we have

$$
\begin{aligned}
P_{i}(u) & =\left(\begin{array}{c}
m \\
i
\end{array}\right) p^{i}(1-p)^{m-i} \\
& \leq(n p)^{-\alpha} \beta^{i},
\end{aligned}
$$

where $\alpha$ is as above and $\beta=e c \log (n p) / \delta$. We have applied (34) and the relation $\left(\begin{array}{c}m \\ i\end{array}\right) \leq\left(\frac{e m}{i}\right)^{i}$ [1] in obtaining the last inequality. 
The event $\overline{A_{k}(u)}$ occurs iff less than $k$ sensors are active in the disc $D_{r}(u)$. Therefore,

$\operatorname{Pr}\left[\overline{A_{k}(u)}\right]=\sum_{i=0}^{k-1} P_{i}(u) \leq \sum_{i=0}^{k-1}(n p)^{-\alpha} \beta^{i} \approx(n p)^{-\alpha} \beta^{k-1}$

and $\operatorname{Pr}\left[A_{k}(u)\right] \geq 1-(n p)^{-\alpha} \beta^{k-1}$. Applying (6) and using the value of $\operatorname{Pr}\left[A_{k}(u)\right]$ in (11), we obtain

$\operatorname{Pr}\left[\bigwedge_{i \in I} A_{k}(i)\right] \geq \exp \left(-5 \ell(n p)^{-\alpha} \beta^{k-1}\right)$.

The exponent of $e$ in (37) should go to 0 for the probability of $k$-coverage to approach 1 . To verify that, we take the logarithm of $\ell(n p)^{-\alpha} \beta^{k-1}$ (the exponent of $e$ in (37) without the " -5 " factor) and expand it using (33) as well as definitions of $\ell, \alpha$ and $\beta$. This yields

$$
\begin{aligned}
& \log \left(\ell(n p)^{-\alpha} \beta^{k-1}\right)=-\phi(n p)-\phi(n p) \sqrt{p \log (n p)} \\
& +\log (\hat{\phi}(n p))+(k-1) \log (e c / \delta)+\sqrt{4 p \pi c \log (n p)} .
\end{aligned}
$$

The right hand side of (38) approaches $-\infty$ forcing the right hand side of (37) to approach 1, which ensures the asymptotic $k$-coverage of all the virtual grid-points in $I$. As mentioned earlier, the $k$-coverage of $C$ and $S$ can be proved similarly. This completes the proof of Claim 2. By Lemma 3.1, we conclude that all the points in the unit square are almost always $k$-covered.

Corollary 4.5. If, for some slowly growing $\phi(n p), p$ and $r$ satisfy

$c(n) \geq 1+\frac{\phi(n p)(1+\sqrt{p \log (n p)})+k \log \log (n p)}{\log (n p)}$

for sufficiently large $n$, then the entire unit square region is almost always $k$-covered.

Note that if a region is not 1 -covered then it is not $k$-covered. Thus, the conditions established in Theorem 4.2 and Corollary 4.3 for non- 1 -coverage also hold for non- $k$-coverage.

\subsection{A paradox}

Our sufficient condition for asymptotic l-coverage (Corollary 4.2) seems to contradict a necessary condition reported in [16]. In Proposition 2.1 of [16], it is claimed that if $p(n) \rightarrow 0$ as $n \rightarrow \infty$, then a necessary condition for asymptotic 1 -coverage (of the unit square) is given by

$\lim \inf _{n \rightarrow \infty} \frac{n p(n) r^{2}(n)}{\log (n)} \geq \frac{1}{\pi}$.
Using our notion of $c(n)$, this (necessary) condition can be rephrased as

$\lim \inf _{n \rightarrow \infty} \frac{c(n) \log (n p)}{\log (n)} \geq 1$

which evidently contradicts our Corollary 4.2. For instance, if we let $p=1 / \sqrt{n}$ and $r^{2}=\log (n) /(1.2 \pi \sqrt{n})$, then $c(n)=$ $\log n /(1.2 \log (n p))$, which approaches infinity; while lim $c(n) \log (n p) / \log n<1$. By Corollary 4.2, the unit square region $i$ almost always 1 -covered; while by Proposition 2.1 of [16], it is not. They cannot be both true. To resolve this paradox, we wish to point out an unproven claim in the proof of Proposition 2.1 in [16]. ${ }^{3}$ Thus, when $p \rightarrow 0$, the necessity of (40) lacks a proof. We established a simple necessary condition in Corollary 4.4 .

In another proposition in [16], it is claimed that asymptotic connectivity does not imply asymptotic coverage. Proposition 4.1 [16], together with Proposition 2.1 (the condition (40) above), was used to support this claim. Now that Proposition 2.1 [16] remains unproven, the question of whether asymptotic connectivity implies asymptotic coverage remains open. ${ }^{4}$

\section{Uniform distribution}

Under uniform distribution, $n$ nodes are distributed uniformly over the square region of unit area as illustrated in Fig. 5. Under this distribution, each node has an equal likelihood of being at any location in the region; and the probability of a given node being in any subregion of area $R$ is $R$.

The proofs for uniform distribution differ from those used in Section 4 for the grid distribution in the following ways:

1. The proofs will be simpler for uniform distribution - and so will the obtained conditions - than for the grid deployment. This is because under the uniform distribution we

\footnotetext{
${ }^{3}$ In the proof of Proposition 2.1, it is shown that the following, (7) in [16], is a necessary condition for asymptotic 1 -coverage:

$4 \bar{c}(n) \log (n) n^{\bar{c}(n) \pi p \theta(p)-1} \stackrel{n \rightarrow \infty}{\longrightarrow} \infty$

where $\bar{c}(n)=n r^{2}(n) / \log (n)$ and $\theta(p)=-\log (1-p) / p$. It is then claimed that (42) holds only if $\bar{c}(n) \pi p \theta(p) \geq 1$. The latter is shown to hold only if (40) is satisfied; and, therefore, it is concluded that (40) is a necessary condition for 1-coverage. Unfortunately, the above claim is not always true. For instance, if we take $p(n)=1 / \sqrt{n}$ and $r^{2}=\log (n) /(1.2 \pi \sqrt{n})$ (and thus $\bar{c}(n)=\sqrt{n} /(1.2 \pi)$ ), then the condition in (42) holds, but $\bar{c}(n) \pi p \theta(p)<1$.

${ }^{4}$ Proposition 4.1 [16] states that the sensor network is almost always connected if $n p e^{\frac{-n p \pi r^{2}}{2}} \stackrel{n \rightarrow \infty}{\longrightarrow} 0$, which in our notation is equivalent to $\lim _{n \rightarrow \infty} c(n)>2$. Since this condition is consistent with our sufficient condition for coverage (Corollary 4.1) for sufficiently large $n$, the claim that asymptotic connectivity does not imply asymptotic coverage remains open.
} 


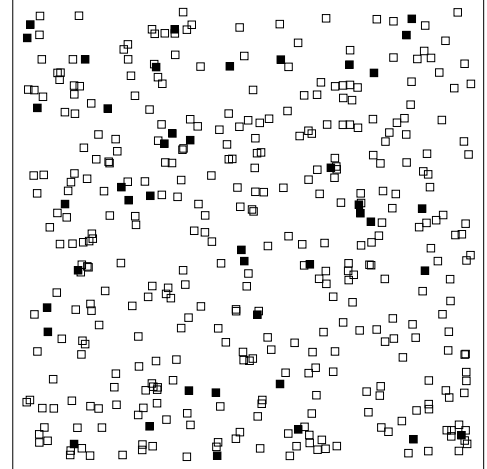

Fig. 5 Random Uniform deployment of sensors on a unit square with $n=400$ and $p=0.1$. The filled squares represent active sensors

do not have to estimate the number of nodes falling in a disc $D_{r}(u)$ (see Remark 2.1).

2. For uniform distribution, the event of coverage (or noncoverage) of a point $u$ in the region is dependent on the event of coverage (or non-coverage) of not only the points located within a distance of $2 r$ from $u$ (so that some sensor can cover both points) but also on the coverage of points more than $2 r$ distance from $u$. This is because if $u$ is not covered, then the probability of coverage of a point $v$ located more than $2 r$ distance from $u$ increases as now all $n$ sensors have a greater likelihood of being in the sensing range of $v$, than if one or more of these sensors was covering $u$. This observation has at least two implications-(1) Janson's Inequality, which was used in the proof of Theorem 4.3 can no longer be used to prove the conditions for almost always $k$-coverage in case of uniform distribution, and (2) establishing that $\Delta=o\left(\mathbb{E}[X]^{2}\right)$, as was done in the proof of Theorem 4.2, is not enough to prove the conditions for almost always non-coverage in case of uniform distribution.

We use the same virtual grid $L$ as introduced in Section 3 . As in the case of grid deployment, showing $L$ almost always covered is sufficient to guarantee the almost always coverage of the entire region. As before, let $c(n)=n p \pi r^{2} / \log (n p)$.

Theorem 5.1. Let $n$ sensors be deployed uniformly over a unit square region. If, for some slowly growing $\phi(n p), p$ and $r$ satisfy

$c(n)=1+\frac{\phi(n p)+k \log \log (n p)}{\log (n p)}$

for sufficiently large $n$, then the unit square region is almost always $k$-covered.

Proof: The proof is similar to that of Theorem 4.3. The key part in that proof is Claim 2. Here, we prove a corresponding claim: If $p$ and $r$ satisfy

$c(n)=1+\frac{\phi(n p)+k \log \log (n p)}{\log (n p)}$

then $L$ is $k$-covered, where, as before, $L$ contains $\ell$ grid points, with $\ell=n p \hat{\phi}(n p) \log (n p)$ and $\hat{\phi}(n p)=\Theta(\phi(n p)$.

Again, we only prove the claim for $I$. Let $A(u), A_{k}(u)$, $N_{r}(u), P_{i}(u)$ and $\beta$ assume the same definition as in the proofs of Theorems 4.1 and 4.3. For any $u \in I$, with probability $p \pi r^{2}$ each individual sensor falls in $D_{r}(u)$ and is active. Therefore, corresponding to (34) and (35), we have

$P_{0}(u)=\operatorname{Pr}[\overline{A(u)}]=\left(1-p \pi r^{2}\right)^{n} \leq(n p)^{-c}$

and, for $i \geq 1$,

$P_{i}(u) \leq(n p)^{-c} \beta^{i}$.

Substituting these values in (36) yields

$\operatorname{Pr}\left[\overline{A_{k}(u)}\right] \leq(n p)^{-c} \beta^{k-1}$.

Similar to the definition of $X$ in the proof of Theorem 4.2, let $X_{k}=X_{k}(1)+X_{k}(2)+\cdots+X_{l}(\ell)$. Here, $X_{k}(i)$ is an indicator random variable assuming a value of 1 if the virtual grid point $i$ is not $k$-covered, and 0 otherwise. Now, $\mathbb{E}\left[X_{k}(u)\right]=\operatorname{Pr}\left[\overline{A_{k}(u)}\right] \leq(n p)^{-c} \beta^{k-1}$, by using (45). Therefore, $\mathbb{E}\left[X_{k}\right] \leq \ell(n p)^{-c} \beta^{k-1}$. Now, we will use a different approach to show $\operatorname{Pr}\left[\bigwedge_{i \in I} A_{k}(i)\right] \rightarrow 1$ than that followed in the proof of Theorem 4.3, where we used Janson's Inequality [1, Theorem 8.1.1]. Since $X_{k}$ is a nonnegative integral valued random variable, $\operatorname{Pr}\left[X_{k}>0\right] \leq \mathbb{E}\left[X_{k}\right]$, and therefore, we have

$$
\begin{aligned}
\operatorname{Pr}\left[\bigwedge_{i \in I} A_{k}(i)\right] & =1-\operatorname{Pr}\left[X_{k}>0\right] \\
& \geq 1-\left(\ell(n p)^{-c} \beta^{k-1}\right) .
\end{aligned}
$$

Now, corresponding to (38), we have (by using (44))

$$
\begin{aligned}
& \log \left(\ell(n p)^{-c} \beta^{k-1}\right) \\
& =-\phi(n p)+\log (\hat{\phi}(n p))+(k-1) \log (e c / \delta) .
\end{aligned}
$$

The right hand side of (49) approaches $-\infty$ forcing the right hand side of (48) to approach 1, which implies that all the grid-points on the virtual are almost always covered. Similar to Theorem 4.3, we conclude with the help of Lemma 3.1 that all the points in the unit square region are almost always covered.

Corollary 5.1. Let $n$ sensors be deployed uniformly over a unit square region. If, for some slowly growing $\phi(n p), p$ and 
$r$ satisfy

$c(n) \geq 1+\frac{\phi(n p)+k \log \log (n p)}{\log (n p)}$

for sufficiently large $n$, then the entire unit square region is almost always $k$-covered.

Theorem 5.2. Let $n$ sensors be deployed uniformly over a unit square region, and assume $\lim _{n \rightarrow \infty} p r^{2}=0$. If, for some slowly growing $\phi(n p), p$ and $r$ satisfy

$c(n)=1-\frac{\phi(n p)+\log \log (n p)}{\log (n p)}$

for sufficiently large $n$, then the unit square region is almost always not 1-covered.

Proof: Let $A(u)$ and $X$ be as defined in the proof of Theorem 4.2. For points $u \in I$, we observe from (45) that

$\operatorname{Pr}[\overline{A(u)}]=p_{0}(u)=\left(1-p \pi r^{2}\right)^{n} \approx(n p)^{-c}$.

Using this value of $\operatorname{Pr}[\overline{A(u)}]$ in (23), the right hand side of (23) remains the same and so $\mathbb{E}[X] \rightarrow \infty$ as $n \rightarrow \infty$.

We will use Corollary 4.3.2 from [1] in place of its Corollary 4.3.4 that was used in the proof of Theorem 4.2. Corollary 4.3.2 states that if $\operatorname{Var}[X]=o\left(\mathbb{E}[X]^{2}\right)$, then $X>$ 0 almost always. Since $X$ is a sum of indicator random variables, we can write

$$
\begin{aligned}
\operatorname{Var}[X] \leq \mathbb{E}[X] & +\sum_{i \neq j} \operatorname{Cov}\left[X_{i}, X_{j}\right] \\
=\mathbb{E}[X] & +\sum_{d(i, j) \leq 2 r} \operatorname{Cov}\left[X_{i}, X_{j}\right] \\
& +\sum_{d(i, j)>2 r} \operatorname{Cov}\left[X_{i}, X_{j}\right]
\end{aligned}
$$

Clearly, $\mathbb{E}[X]=o\left(\mathbb{E}[X]^{2}\right)$. As was shown in the proof of Corollary 4.3.4 in [1], $\sum_{i \neq j} \operatorname{Cov}\left[X_{i}, X_{j}\right] \leq \Delta$. We will apply this inequality to the second term in (53) to show that for the case of $d(i, j) \leq 2 r, \Delta=o\left(\mathbb{E}[X]^{2}\right)$ and consequently $\sum_{d(i, j) \leq 2 r} \operatorname{Cov}\left[X_{i}, X_{j}\right]=o\left(\mathbb{E}[X]^{2}\right)$.

Using the value of $\operatorname{Pr}[\overline{A(u)}]$ from (45), we have corresponding to (25) the following:

$P_{r}=\operatorname{Pr}[\overline{A(u)} \wedge \overline{A(v)} \mid 0<d(u, v)<r] \leq(n p)^{-c}$.

For $P_{2 r}$, we have the same inequality as (26). Using values from (54) and (26) in (27), we obtain

$\Delta \leq \frac{\ell}{2}\left(\ell_{r}(n p)^{-c}+\ell_{2 r}(n p)^{-4 c / 3}\right)$.
Using the upper bound on $\Delta$ from (55) and the lower bound on $\mathbb{E}[X]$ from (23) (as was shown earlier it holds for the uniform distribution as well), in (28), we obtain

$\frac{\Delta}{\mathbb{E}[X]^{2}} \leq \frac{\ell_{r}(n p)^{-c}+\ell_{2 r}(n p)^{-4 c / 3}}{2 \ell(n p)^{-2 c}}$.

Thus, using (51), we obtain

$\log \left(\frac{\log (n p)(n p)^{-c}}{(n p)^{-1-2 c}}\right)=-\phi(n p)$.

in place of (30). The right hand side of (57) approaches $-\infty$. Therefore, for the case of $d(i, j) \leq 2 r, \Delta=o\left(\mathbb{E}[X]^{2}\right)$ and consequently $\sum_{d(i, j) \leq 2 r} \operatorname{Cov}\left[X_{i}, X_{j}\right]=o\left(\mathbb{E}[X]^{2}\right)$. What remains to be shown for $\operatorname{Var}[X]=o\left(\mathbb{E}[X]^{2}\right)$ to hold is to show that $\sum_{d(i, j)>2 r} \operatorname{Cov}\left[X_{i}, X_{j}\right]=o\left(\mathbb{E}[X]^{2}\right)$, which we now prove.

We notice that

$$
\begin{aligned}
\operatorname{Cov}\left[X_{i}, X_{j}\right] & =\mathbb{E}\left[X_{i} X_{j}\right]-\mathbb{E}\left[X_{i}\right] \mathbb{E}\left[X_{j}\right] \\
& =\operatorname{Pr}[\overline{A(i)} \wedge \overline{A(j)}]-\operatorname{Pr}[\overline{A(i)}] \operatorname{Pr}[\overline{A(j)}] .
\end{aligned}
$$

Applying the equality in (58) to the case of $d(i, j)>2 r$ and using (52), we get

$$
\begin{aligned}
\operatorname{Cov}\left[X_{i}, X_{j}\right] & =\operatorname{Pr}[\overline{A(i)} \wedge \overline{A(j)}]-\operatorname{Pr}[\overline{A(i)}] \operatorname{Pr}[\overline{A(j)}] \\
& =\left(1-2 p \pi r^{2}\right)^{n}-\left(1-p \pi r^{2}\right)^{2 n}<0 .
\end{aligned}
$$

Since $\operatorname{Cov}\left[X_{i}, X_{j}\right]<0$ for $d(i, j)>2 r$, and $\mathbb{E}[X] \rightarrow$ $\infty, \sum_{d(i, j)>2 r} \operatorname{Cov}\left[X_{i}, X_{j}\right]=o\left(\mathbb{E}[X]^{2}\right)$. This proves that $\operatorname{Var}[X]=o\left(\mathbb{E}[X]^{2}\right)$, and we conclude from Corollary 4.3.2 in [1] that if (51) holds, then there almost always exists a non-covered point in the region. In fact, from Corollary 4.3.3 in [1], we can even conclude that almost always $X \approx \mathbb{E}[X]$. This completes the proof.

Corollary 5.2. Let $n$ sensors be deployed uniformly over a unit square region, and assume $\lim _{n \rightarrow \infty} p r^{2}=0$. If, for some slowly growing $\phi(n p), p$ and $r$ satisfy

$c(n) \leq 1-\frac{\phi(n p)+\log \log (n p)}{\log (n p)}$

for sufficiently large $n$, then the unit square region is almost always not 1-covered.

\section{Poisson distribution}

In the Poisson model of deployment, number of nodes deployed follows Poisson distribution with rate $n$. The actual 
number of nodes deployed need not be $n$. Following the proofs for uniform distribution developed in Section 5, similar results for Poisson distribution can be easily derived. Therefore, in this section we only state the results, and focus more on showing the equivalence of our condition and that in [19] for $k$-coverage under Poisson distribution. We first state our results.

Theorem 6.1. Let sensors be deployed over a unit square region according to a Poisson process with rate $n$. If, for some slowly growing $\phi(n p), p$ and $r$ satisfy

$c(n)=1+\frac{\phi(n p)+k \log \log (n p)}{\log (n p)}$

for sufficiently large $n$, then the entire unit square region is almost always $k$-covered.

Theorem 6.2. Let sensors be deployed over a unit square region according to a Poisson process with rate $n$. If, for some slowly growing $\phi(n p), p$ and $r$ satisfy

$c(n)=1-\frac{\phi(n p)+\log \log (n p)}{\log (n p)}$

for sufficiently large $n$, then the unit square region is almost always not 1-covered.

The conditions in the corresponding corollaries are the same as in Corollary 5.1 and Corollary 5.2.

We now show how to translate the results developed for the model considered in this paper to the model considered in [19] and vice versa. In this paper, we model the region as a unit square with $n$ as the rate of Poisson distribution and $r$ the sensing radius. (We will assume $p=1$, so that $n p=n$.) In [19], the region was modeled as a square with side length $\ell$, each sensing disk had unit area, and the rate of Poisson distribution was $\lambda$.

If we scale the side length of square from $\ell$ to unity, then the rate of Poisson distribution becomes $\ell^{2} \lambda$. Since $n$ is the rate of Poisson distribution in a unit square in our model,

$n=\lambda \ell^{2}$,

and since the original rate, $\lambda$, indicated the expected number of sensors in a sensing disk, we obtain

$n \pi r^{2}=\lambda$

We now show that (61) (with $p=1$ ) is essentially the same as the condition for $k$-coverage in [19], which for con- venience is restated below:

$\lambda=\log \left(\ell^{2}\right)+(k+1) \log \log \left(\ell^{2}\right)+c(\ell)$,

where $c(\ell) \rightarrow \infty$.

Substituting the value of $c(n)$ from (1) in (61) and setting $p=1$, we obtain

$n \pi r^{2}=\log (n)+k \log \log (n)+\phi(n)$,

which can be restated using (63) and (64) as

$$
\begin{aligned}
\lambda= & \log \left(\lambda \ell^{2}\right)+k \log \log \left(\lambda \ell^{2}\right)+\phi\left(\lambda \ell^{2}\right) \\
= & \log \left(\ell^{2}\right)+(k+1) \log \log \left(\ell^{2}\right) \\
& +\phi\left(\lambda \ell^{2}\right)+(k+1)\left[\log \log \left(\lambda \ell^{2}\right)-\log \log \left(\ell^{2}\right)\right]
\end{aligned}
$$

By observing from (66) that $\log \left(\ell^{2}\right) \leq \lambda=O\left(\log \left(\ell^{2}\right)\right)$, (66) can be transformed to (65).

Conversely, (65) can be transformed to (61) as follows: if $c(\ell)=O\left(\log \log \log \left(\ell^{2}\right)\right)$. Substituting $\ell^{2}=n / \lambda$ and $\lambda=$ $n \pi r^{2}$ from (63) and (64) in (65) yields

$$
\begin{aligned}
n \pi r^{2}= & \log (n)-\log (\lambda)+\log \log \left(\ell^{2}\right)+k \log \log \left(\ell^{2}\right)+c(\ell) \\
= & \log (n)+k \log \log (n)+[c(\ell) \\
& \left.+k \log \log \left(\ell^{2}\right)-k \log \log \left(\ell^{2} \log \left(\ell^{2}\right)\right)\right] .
\end{aligned}
$$

Here we have used (64), (65), and the fact that $\lambda \ll n$. If $c(\ell)=O\left(\log \log \log \left(\ell^{2}\right)\right)$, then (67) is same as (65).

\section{Simulation}

In this section, we describe how the number of sensors needed to achieve $k$-coverage varies with $k$ in analysis and in simulation. We focus on grid deployment as the results for other deployments are similar.

We use $p=0.2$, which means that the network is desired to last five times the lifetime of an individual sensor. We use $r=0.04$, which implies that the side of the square region of deployment is 25 times the sensing radius.

We first describe the procedure we follow to compute the number of sensors required to achieve $k$-coverage in analysis, which we denote by $n^{a}(k, p, r)$, for a given value of $k, p$, and $r$. The value of $n^{a}(k, p, r)$ is given by

$\min _{n}\left\{\frac{n p \pi r^{2}}{\log (n p)} \geq \frac{1+\phi(n p)(1+\sqrt{p \log (n p)})+k \log \log (n p)}{\log (n p)}\right\}$.

One issue here is what function to use for $\phi(n p)$. We discuss this issue when describing the simulation results later in this section. 
We now describe the procedure to determine the number of sensors required to achieve $k$-coverage in simulation, which we denote by $n^{s}(k, p, r)$, for a given value of $k, p$, and $r$. We consider the deployment of $n$ sensors in a grid for a particular value of $n$. In one iteration, we activate each of these $n$ sensors with probability $p$. We then compute the number of virtual grid points (of which there are $\ell=n p \phi(n p) \log (n p)$ of them) $k$-covered by the active sensors with a sensing radius of $r^{\prime}=$ $r-1 / \sqrt{2 \ell}$. If all points on the virtual grid are covered in this iteration, we conclude by Lemma 3.1, that all points in the square are $k$-covered with the actual sensing radius of $r$ in this interation. We perform 100 iterations for each value of $n$. The probability of $k$ coverage is then approximated as the fraction of interations in which all points in the square region were $k$-covered.

In simulation, we vary the number of sensors $n$, starting with a value close to $n^{a}(k, p, r)$ and find the minimum value of $n$ for which the probability of $k$-coverage is 1 . This is the value of $n^{s}(k, p, r)$.

Figure 6 shows the values of $n^{a}(k, p, r)$ and $n^{s}(k, p, r)$. We make several observations from this figure. First, we see that the results of analysis closely matches the results of simulation. Second, there is a sharp increase in the number of nodes needed to achieve 1 -coverage from 0 -coverage. But, the number of nodes needed to achieve 2-coverage is only marginally more than that needed for 1 -coverage. From the simulation plot in Fig. 6, we see that 15,600 sensors are needed to achieve $l$-coverage. But, deploying only 1,275 more sensors provides 2 -coverage. Third, we observe that as the value of $k$ increases, the curve of $n^{a}(k, p, r)$ becomes flatter, which indicates that the additional number of nodes needed to achieve an additional degree of coverage will decrease with increasing value of $k$.

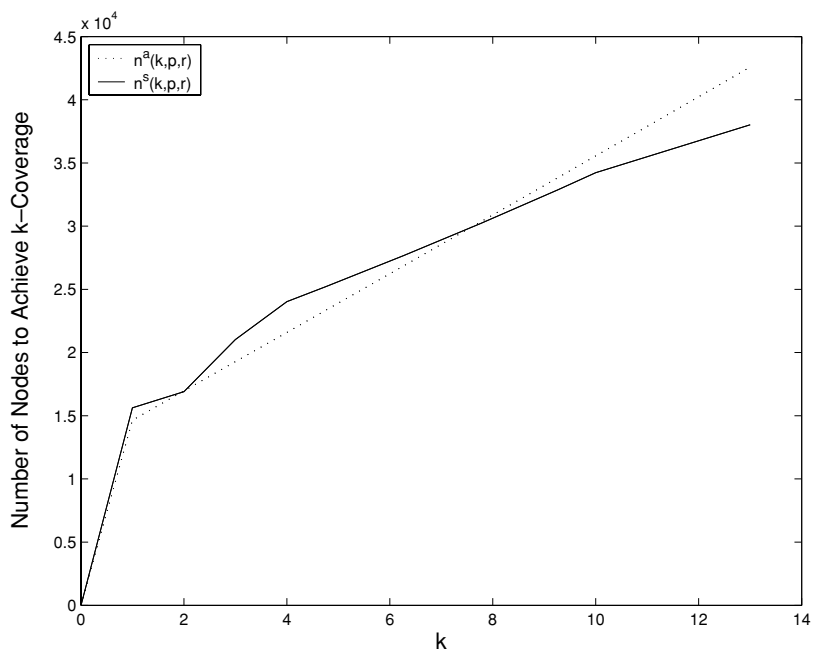

Fig. 6 Number of sensor nodes needed to achieve $k$-coverage for $p=$ 0.2 and $r=0.04$ as $k$ is varied
Finally, observe that as the value of $k$ increases, the predictions of analysis in terms of the number of nodes needed to achieve $k$-coverage changes from conservative to liberal. It means to match the simulation results more closely for values of $k$ larger than 10 , we need to choose a more appropriate value of $\phi(n p)$. It is the value of $\phi(n p)$ that largely determines how closely the analysis will match simulation results. Here, we chose $(\log \log (n p))^{0.99}$ as the value of $\phi(n p)$, which provided a close match with simulation results for $k \leq 10$. If the value of $k$ is larger than 10 , or if the value of $r$ is smaller than 0.04 , or if the value of $p$ is smaller than 0.2 , a slower growing function will be a more appropriate choice for $\phi(n p)$.

We end this section by discussing the effect of varying $p$ on the number of nodes needed to achieve $k$ coverage for a fixed value of $k$, and $r$. Notice that in all the conditions for coverage, $n$ and $p$ appear together so that we can use $n^{\prime}=n p$ in all the conditions, as long as the assumptions on $p$ are met. It means that the conditions for coverage depend on $n^{\prime}=n * p .{ }^{5}$ Further, for a given value of $k$ and $r$, the value of $n^{\prime}$ that provides $k$-coverage with high probability is uniquely determined. The value of $n$ then can be uniquely determined, as well, given a value of $p$ by computing $n=n^{\prime} / p$. For the example deployment considered in this section, 1 -coverage is achieved at $n=\left(n^{a}(k, p, r)\right)=14,651$, when $p=0.2$. If $p=0.1,1$-coverage will be achieved at $n=29,302$.

\section{Conclusion}

In this paper, we considered the fundamental problem of determining the appropriate number of sensors that are enough to provide $k$-coverage of a region when sensors are allowed to sleep most of their lifetime, which in turn is necessary to make sensor networks long-lived. We derived critical conditions for these kinds of sensor networks. We showed that the conditions for deterministic deployment are similar to the conditions for random deployments. At the same time, the minor difference in the conditions for these two types of deployments indicates that the deterministic model with RIS sleeping is not exactly the same as the random deployment models with RIS sleeping. We also showed that our analytical values match closely with simulation results, supporting our claim that our results can be used in real-life applications for determining the appropriate number of sensors to be deployed, given the area to be covered, the sensing radius of the sensors, and the desired network lifetime. Fi-

\footnotetext{
${ }^{5}$ In the conditions for coverage for uniform and Poisson distribution, every occurrence of $n$ and $p$ always occur together as $n * p$, which can be easily replaced with $n^{\prime}$. In the grid deployment case, there is an error term $\sqrt{p \log (n p)}$. Here $p$ appears alone. This term, however, has only a marginal effect on the conclusion of this discussion.
} 
nally, the approach of independent sleeping proposed in our RIS scheme makes the sentry selection task (to ensure $k$ coverage) energy-efficient and light-weight because sensors do not require any interaction with their neighbors.

Acknowledgments The authors would like to thank Anish Arora and Marc E. Posner from the Ohio State University, Jason Hallstrom from Clemson University, and Ho Woo Lee from Sung Kyun Kwan University, South Korea for their valuable comments and feedback on an earlier draft of this paper. Santosh Kumar's work was partially supported by DARPA contract OSU-RF \#F33615-01-C-190. József Balogh's work was partially supported by National Science Foundation grant \# DMS 0302804 and OTKA grant 049398.

\section{References}

1. N. Alon and J.H. Spencer, The probabilistic method, (John Wiley \& Sons, 2000).

2. A. Arora et al., Line in the sand: A wireless sensor network for target detection, classification, and tracking. Technical report, OSUCISRC-12/03-TR71, Ohio State University (2003).

3. C. Bettstetter, On the minimum node degree and connectivity of a wireless multihop network, in: Proceedings of ACM MobiHoc, Lausanne Switzerland (2002) pp. 80-91.

4. B. Bollobás, Random Graphs, (Cambridge University Press 2001).

5. Crossbow, Power management and batteries, Application Notes, Available at http://www.xbow.com/Support/appnotes.htm (2004).

6. P. Gupta and P.R. Kumar, Critical power for asymptotic connectivity in wireless networks, in: IEEE 37th Conference on Decision and Control. Tampa, FL (1998) pp. 1106-1110.

7. D.L. Hall and J. Llinas, Handbook of Multisensor Data Fusion, (CRC Press 2001).

8. P. Hall, Introduction to the Theory of Coverage Processes, (John Wiley \& Sons, 1988).

9. T. He, S. Krishnamurthy, J.A. Stankovic, T. Abdelzaher, L. Luo, R. Stoleru, T. Yan and L. Gu, Energy-efficient surveillance system using wireless sensor networks, in: Proceedings of ACM Mobisys, Boston, MA (2004).

10. M. Horton, D.E. Culler, K. Pister, J. Hill, R. Szewczyk and A. Woo, The commercialization of microsensor motes, in: Sensors volume Vol. 19, No. 4, (April 2002) pp. 40-48.

11. C. Huang and Y. Tseng, The coverage problem in a wireless sensor network, in: Proceedings of WSNA, San Diego, CA (2003) pp. 115121.

12. J. Hui, Z. Ren and B.H. Krogh, Sentry-based power management in wireless sensor networks, in: Proceedings of IPSN, Palo Alto, CA (2003) pp. 458-472.

13. X. Li, P. Wan, Y. Wang and C. Yi, Fault tolerant deployment and topology control in wireless networks, in: Proceedings of ACM MobiHoc, Annapolis, MD (2003) pp. 117-128.

14. R. Meester and R. Roy, Continuum Percolation, Cambridge University Press (1996).

15. S. M. Ross, Introduction to Probability Models, Academic Press (2000).

16. S. Shakkottai, R. Srikant and N.B. Shroff, Unreliable sensor grids: coverage, connectivity and diameter, in: Proceedings of IEEE INFOCOM vol. 2, San Francisco, CA (2003) pp. 1073-1083.
17. R. Szewczyk, J. Polastre, A.M. Mainwaring and D.E. Culler, Lessons from a sensor network expedition, in: EWSN, Berlin, Germany (2004).

18. X. Wang, G. Xing, Y. Zhang, C. Lu, R. Pless and C. Gill, Integrated coverage and connectivity configuration in wireless sensor networks, in; Proceedings of ACM Sensys, Los Angeles, CA (2003) pp. 28-39.

19. H. Zhang and J. Hou, On deriving the upper bound of $\alpha$-lifetime for large sensor networks, in: Proceedings of ACM MobiHoc, Tokyo, Japan (2004) pp. 121-132.

20. J. Zhao and R. Govindan, Understanding packet delivery performance in dense wireless sensor networks, in: Proceedings of ACM Sensys, Los Angeles, CA (2003) pp. 1-13.

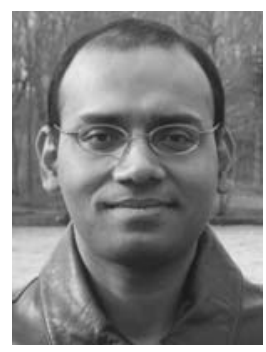

Santosh Kumar is an Assistant Professor in the department of Computer Science at the University of Memphis. He received his Ph.D. in Computer Science and Engineering from the Ohio State University in 2006, where he was the SBC Presidential Fellow in 2006. He also received an M.S. in Computer and Information Science from the Ohio State University in 2002. He received a B.Tech. in Computer Science and Engineering from the Institute of Technology at Banaras Hindu University (IT-BHU) in 1998. His current research focuses on the issues of coverage and connectivity in wireless sensor networks.

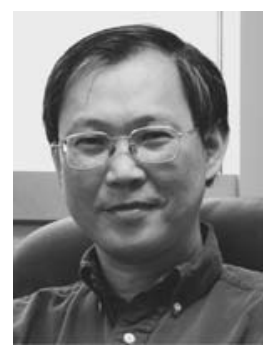

Ten-Hwang Lai is a Professor of Computer Science and Engineering at the Ohio State University. A pioneer of Zen Networking, he is interested in the art of applying Zen to the teaching and research of protocol design. He served as the program chair of ICPP'98, the general chair of ICPP' 00 , the program co-chair of ICDCS'04, and, recently, the general chair of ICDCS'05. He is/was an editor of IEEE Transactions on Parallel and Distributed Systems (TPDS), ACM/Springer Wireless Networks, Academia Sinica's Journal of Information Science and Engineering (JISE), and International Journal of Ad Hoc and Ubiquitous Computing. 


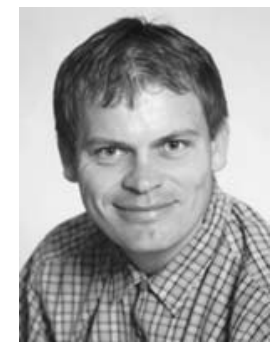

Illinois, he held post doctoral positions at AT\&T (2001-2002), Princeton (2002), and the Ohio State University (2002-2005). His current research interests include Probabilistic Methods in Discrete Mathematics and Extremal Graph Theory.

József Balogh is an Assistant Professor of Mathematics at the University of Illinois at Urbana-Champaign. He received an M.S. from Szeged University, Hungary in 1995 and a Ph.D. in Mathematics from the University of Memphis in 2001. Before joining the University of 